\title{
Losartan:
}

\section{il valore clinico ed economico}

Lorenzo Pradelli*, Mario Eandi*

REVIEW

\begin{abstract}
Losartan is an angiotensin II receptor antagonist (ARB) that has been proven effective and well tolerated in the treatment of hypertension and in the reduction of global cardiovascular risk, but is more expensive than many alternatives. The present paper resumes its main pharmacological and clinical features, as a basis for analyzing its economical value.

Despite the universally accepted relevance of good blood pressure control, hypertension is still undertreated and poorly controlled. Among the main reasons for this, poor adherence to therapeutic regimens plays a relevant role.

Losartan and ARBs have been shown to be associated with compliance rates superior to those observed with any other antihypertensive drug class, due to an adverse events incidence similar to placebo and scarcity of relevant pharmacological interactions, and can therefor improve the cost-effectiveness of hypertension treatment.

Several trials have indicated that losartan determines clinical benefits that go beyond its haemodynamic effect, such as renal protection, reduced incidence of stroke and cognitive improvement. The addition of losartan to conventional hypertensive therapy in diabetic patients with nephropathy has been estimated to induce net health cost savings in different settings, a relevant result, as very few medical interventions have demonstrated such a potential.

To understand the real economical value of losartan, its drug acquisition costs have to be balanced against the benefits deriving from antihypertensive effectiveness, high compliance and non-haemodynamic target organ protection.
\end{abstract}

Farmeconomia e percorsi terapeutici 2004; 5 (1): 13-23

\section{INTRODUZIONE}

Le conseguenze economiche della scelta di un trattamento farmacologico non dipendono solamente dai costi farmaceutici del prodotto scelto, ma ancor più dalle implicazioni cliniche che il suo uso comporta. Questo concetto base della farmacoeconomia, apparentemente banale, non è invece ancora entrato completamente nel patrimonio culturale della classe medica, che spesso, di fronte ad un trattamento di costo più elevato delle alternative disponibili ma di efficacia differente, non apprezza appieno i potenziali risparmi economici associati ai vantaggi clinici, più prontamente individuati e riconosciuti.

L'introduzione degli antagonisti dei recettori dell' angiotensina II (ARB o sartani) rappresenta un ulteriore opzione a disposizione del medico nella cura e nella prevenzione delle malattie cardiovascolari, prima causa di mortalità e invalidità nei paesi sviluppati, e in tal senso rappresenta sicuramente un progresso medico. La disponibilità di un farmaco preventivo efficace e ben tollerato, in particolare se applicato a soggetti portatori di alto rischio di incorrere in eventi patologici ad alto costo clinico e sociale, ha inoltre la potenzialità di ridurre le conseguenze economiche della patologia cardiovascolare.

Obiettivo del presente lavoro è delineare un profilo farmacologico clinico del losartan, per poi evidenziare le ricadute economiche delle caratteristiche farmacologiche e cliniche della molecola, attraverso una revisione di letteratura selezionata e in considerazione delle particolarità della malattia ipertensiva.

\section{FARMACOLOGIA}

Losartan è il primo inibitore dei recettori dell' angiotensina II (ATII) introdotto sul mercato. Ė stato sviluppato in considerazione del
*Farmacologia Clinica, Università di Torino 
ruolo chiave che il sistema reninaangiotensina-aldosterone esercita nel controllo pressorio e nello sviluppo del danno d'organo associato, come alternativa all'inibizione della formazione dell'angiotensina II determinata dagli ACE-inibitori. I dati sperimentali indicano la presenza di almeno due tipi di recettori, denominati di tipo 1 e 2 (AT1 e AT2), ampiamente distribuiti nei tessuti umani, in cui esplicano azioni funzionalmente antagonistiche tra loro. I recettori AT1 sono quelli maggiormente rappresentati nei tessuti adulti e mediano la maggior parte delle azioni conosciute dell'angiotensina II, nonostante questa abbia affinità paragonabile per i due tipi recettoriali. Losartan, come gli altri ARB sviluppati successivamente, agisce selettivamente sui recettori del sottotipo 1 , sui quali esercita antagonismo competitivo senza effetti agonisti, influenzando in maniera minore o trascurabile (> 10,000 meno affine) gli altri. Le differenze farmacologiche rispetto agli ACE-inibitori sono legate proprio al blocco selettivo di questi recettori, responsabili degli effetti emodinamici e locali dell'ATII, con mantenimento delle funzioni svolte dai recettori AT2, normalmente di tipo antagonistico rispetto agli AT1, almeno per quanto è stato chiarito finora. Questo schema è evidentemente troppo semplicistico e generale per poter spiegare in maniera soddisfacente l'insieme delle funzioni regolatorie del sistema renina-angiotensina-aldosterone (tono vascolare, bilancio elettrolitico, rimodellamento del miocardio, trofismo vascolare, funzione endoteliale ecc.), ma può servire in prima approssimazione per rendere conto delle differenze osservabili tra le due classi farmacologiche, riassunte in Tabella 1.

Dopo somministrazione per via orale, il $25 \%$ circa della dose di losartan viene assorbito a livello sistemico, producendo le concentrazioni di picco plasmatico all'incirca dopo un'ora. Una consistente quota del farmaco assorbito viene trasformata nel metabolita E-3174, che possiede attività di antagonismo AT1 più spiccata del losartan stesso, e viene considerato responsabile della lunga durata dell'azione antipertensiva (fino a 24 ore) che si osserva nella pratica clinica. L'emivita di eliminazione di losartan è di circa 2 ore, quella del metabolita di 4-5; E-3174 ha una cinetica di dissociazione dal recettore molto lenta [1].

\section{EFFICACIACLINICA}

L'efficacia clinica di losartan è stata approfonditamente esplorata in molteplici studi clinici, condotti sia in fase di registrazione che successivamente, di cui riassumiamo i dati più rilevanti. Da un punto di vista teorico, le azioni terapeutiche di losartan possono essere suddivise in due grandi categorie: quelle direttamente dipendenti dalla modulazione dell'emodinamica, come nel trattamento dell'ipertensione e dello scompenso cardiaco, e quelle legate ad altri effetti, consistenti prevalentemente nella protezione del danno d'organo, superiore a quella attesa in base alla mera riduzione della pressione sanguigna.

L'efficacia di losartan nel ridurre la pressione arteriosa è ben documentata, sia quando impiegato come unico agente nei casi di ipertensione lieve e moderata, sia in combinazione ad altri farmaci nei casi insufficientemente controllati in monoterapia.

Nel 1992 Weber [2] ha condotto uno studio controllato vs. placebo su 440 pazienti ambulatoriali con ipertensione di grado lieve o moderato. Le riduzioni pressorie medie registrate dopo 8 settimane di terapia con $50 \mathrm{mg}$ di losartan in monosomministrazione giornaliera sono state di $15 / 12 \mathrm{mmHg}$.

Owens ha trattato soggetti con ipertensione moderata per 6 settimane con il medesimo dosaggio, ottenendo riduzioni medie della pressione diurna di 16,0/9,9 $\mathrm{mmHg}$. Tali riduzioni sono risultate insufficienti a raggiungere l'obiettivo pressorio prefissato (diastolica < $95 \mathrm{mmHg}$ ) in un buon numero di pazienti, successivamente randomizzati in due gruppi di trattamento: in uno di essi il protocollo sperimentale prevedeva l'aumento di dosaggio di losartan a $100 \mathrm{mg} / \mathrm{die}$, nell'altro i soggetti hanno ricevuto losartan $50 \mathrm{mg}$ associato a idroclorotiazide $12,5 \mathrm{mg}$. Quest'ultima opzione è risultata significativamente più efficace del raddoppio del dosaggio di losartan e ha con-

\section{Principali differenze farmacodinamiche tra ARB e ACE-inibitori}

\section{Tabella 1}

Principali differenze farmacodinamiche tra ARB e ACE-inibitori maggior riduzione dell'attivazione dei recettori AT1

attivazione indiretta dei recettori AT2 (per aumento angiotensina II libera)

minor incremento di angiotensina 1-7

nessun aumento dei livelli di altri substrati dell'ACE, tra cui bradichinina e Ac-SDKP 
sentito ulteriori riduzioni della pressione, in media di 10,7/8,4 mmHg [3].

Questi risultati sono in accordo con i dati sperimentali e con l'esperienza clinica della terapia antipertensiva, che generalmente indicano che nella maggioranza dei pazienti, soprattutto in quelli affetti dalle forme più gravi, il controllo pressorio risulta efficace e tollerato più facilmente con regimi polifarmacologici che con l'utilizzo di un singolo principio attivo.

Losartan è efficace anche nella terapia dell'ipertensione diabetica, come dimostrato in vari studi clinici. Lozano ha arruolato 442 pazienti diabetici con ipertensione (PAS $>140 \mathrm{mmHg}$ e/o PAD > $90 \mathrm{mmHg}$ ) e microalbuminuria (30$300 \mathrm{mg} / \mathrm{die}$ ) in uno studio multicentrico e openlabel [4]. I pazienti hanno iniziato la terapia con losartan $50 \mathrm{mg} /$ die per 4 settimane; i pazienti in cui la pressione non era sufficientemente controllata con questo regime sono passati a 100 $\mathrm{mg} /$ die. Se anche questo dosaggio non risultava in grado di portare la pressione al di sotto dell'obiettivo prefissato $(<140 / 90 \mathrm{mmHg})$, il trattamento veniva sostituito con losartan 50 $\mathrm{mg}$ più idroclorotiazide $12,5 \mathrm{mg}$, in unica somministrazione giornaliera. Dopo 6 mesi di studio, la PAS media è scesa da 167 a $137 \mathrm{mmHg}$, la PAD da 96 a $82 \mathrm{mmHg}$ ( $\mathrm{p}<0,001$ per entrambe le variazioni), permettendo il raggiungimento dell'obiettivo pressorio sistolico nel $70 \%$ della coorte ( $23 \%$ dei quali con il diuretico) e di quello diastolico nel $82 \%$ dei pazienti (20\% con diuretico). Oltre al controllo dell'ipertensione, i dati di questo studio rivelano che il trattamento con losartan ha migliorato il controllo glicemico (riduzioni statisticamente significative della glicemia a digiuno e della percentuale di emoglobina glicata) e ridotto l'entità della microalbuminuria (da 115 a 66 mg/24 ore, p < 0,0001), conseguendo la normalizzazione di questo parametro nel $25 \%$ del campione. L'analisi di regressione multipla condotta sui dati ha indicato che solamente il $4 \%$ della variazione dell' escrezione di albumina era attribuibile alle riduzioni di pressione ed emoglobina glicata, suggerendo la presenza di altri meccanismi di protezione renale.

Lo studio ELITE è stato disegnato per confrontare efficacia e sicurezza di losartan e captopril nel trattamento a lungo termine dello scompenso cardiaco congestizio [5]. Sono stati arruolati 350 pazienti ultrasessantacinquenni con disfunzione sistolica (frazione di eiezione $<40 \%$ ) e scompenso di classe II-IV secondo la classificazione NYHA, cui è stata mantenuta la terapia in corso, basata su digitalici e vasodilatatori. Dopo 48 settimane di terapia, il gruppo assegnato all'ARB ha mostrato una riduzione inaspettata ma significativa della mortalità totale. I pazienti trattati con losartan hanno mostrato ridotta mortalità sia cardiovascolare sia da altre cause, risultanti complessivamente in tassi di $4,8 \%$, paragonato a $8,7 \%$ nel gruppo captopril; la differenza deriva in maniera preponderante dalla diversa frequenza di morte improvvisa (1,4\% vs. $3,8 \%$ ). Lo studio non era stato disegnato per individuare differenze in termini di mortalità, ma per paragonare gli effetti dei trattamenti sull'evoluzione della disfunzione renale, risultata sovrapponibile nei pazienti dei due gruppi: per questi motivi, gli stessi ricercatori hanno progettato un nuovo lavoro, ELITE II, per verificare specificamente l'ipotesi che losartan fosse associato a minore mortalità totale e minor tasso di morte improvvisa [6]. I risultati di questo studio non hanno evidenziato differenze significative dei parametri di valutazione tra gruppi. Il complesso dei dati ottenuti nella popolazione dei pazienti con scompenso congestizio ha comunque confermato che losartan possiede efficacia equivalente agli ACE-inibitori, considerati la terapia standard in questi pazienti, e migliore tollerabilità, confermata dal minor numero di abbandoni per eventi avversi nei pazienti losartan, significativo in entrambi gli studi.

Alcuni risultati degli studi ELITE con losartan erano inaspettati, in quanto evidenziavano vantaggi clinici superiori a quelli attesi. In particolare, la protezione degli organi bersaglio - principalmente rene e cuore - non poteva essere ricondotta interamente agli effetti emodinamici del blocco selettivo dei recettori AT1, per cui sono stati ipotizzati meccanismi locali aggiuntivi, come la riduzione del rilascio di catecolamine vasoattive o lo stimolo della circolazione collaterale dovuta all' attività del recettore AT2, ma tali supposizioni necessitano di conferme sperimentali.

Per verificare l'ipotesi che i benefici clinici di losartan nella prevenzione del rischio cardiovascolare superino quelli legati alla riduzione della pressione arteriosa, all'inizio degli anni Novanta è stato progettato lo studio LIFE, in cui sono stati confrontati gli esiti del trattamento con losartan o atenololo in oltre 9000 pazienti ipertesi con evidenza elettrocardiografica di insufficienza ventricolare sinistra (IVS) [7]. I partecipanti, di età compresa tra i 55 e gli 80 anni, sono stati autorizzati a continuare la loro terapia antipertensiva (con eccezione di ACE-inibitori e ARB), cui è stato aggiunto il trattamento sperimentale, titolato per raggiungere l'obiettivo pressorio, prefissato in valori inferiori a 140/90 $\mathrm{mmHg}$. Come end-point primario è stato adottato un parametro composito, costituito dall'incidenza complessiva di decesso, infarto miocardico e ictus cerebrale. A fronte di un 


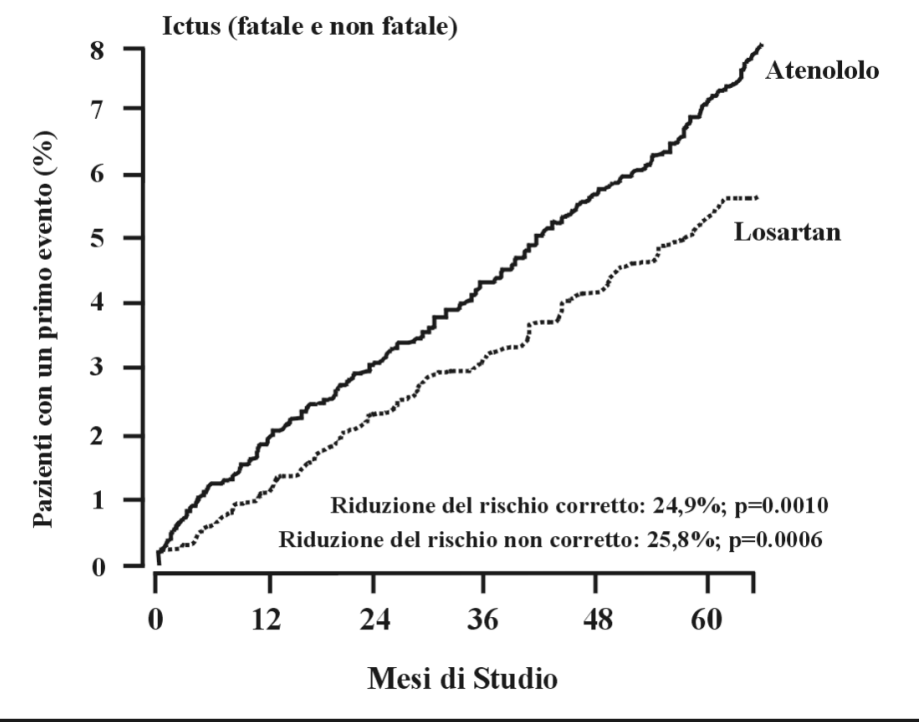

Figura 1

Curve di Kaplan-Meier per ictus fatale e non fatale nello studio LIFE [7]

controllo pressorio pressoché sovrapponibile, per quanto leggermente migliore con losartan (144,1/81,3 vs. 145,4/80,9 mmHg), l'end-point primario si è verificato in maniera significativamente più frequente $(\mathrm{RR}-13 \% ; \mathrm{p}=0,02)$ nei pazienti trattati con il beta-bloccante. Questa differenza è quasi totalmente riconducibile al vantaggio di losartan in termini di prevenzione dell'ictus (5\% vs. 7\%, RR - 25\%; $\mathrm{p}<0,001-$ vedi Figura 1), poiché le altre due componenti del parametro di valutazione adottato (morte cardiovascolare e infarto) non hanno mostrato differenze di incidenza statisticamente significative tra i gruppi di trattamento.

Lo studio ha inoltre rivelato due ulteriori vantaggi del trattamento con losartan: una ri-

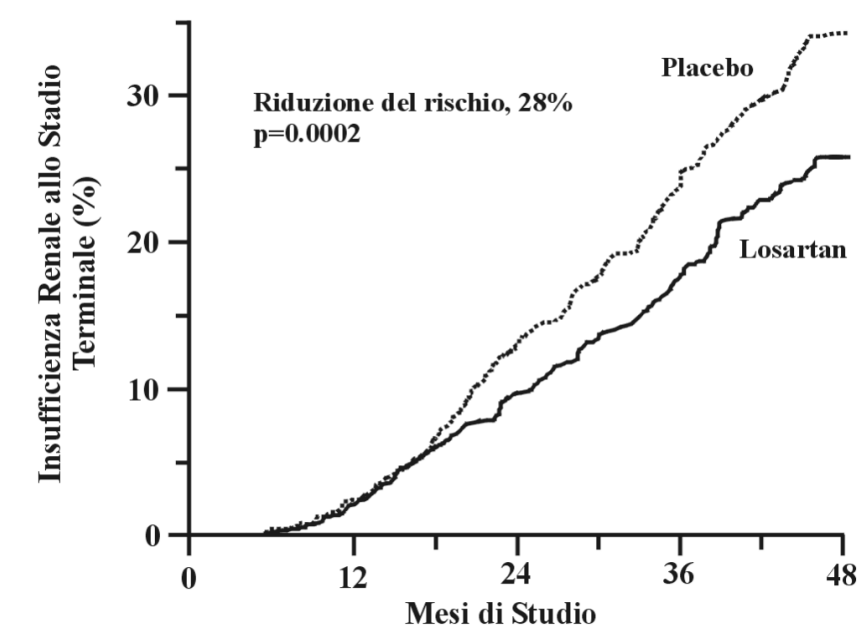

Figura 2

Curve di Kaplan-Meier per insufficienza renale cronica nello studio RENAAL [10] duzione significativamente superiore $(\mathrm{p}<$ $0,0001)$ dell'IVS al termine del periodo di valutazione e la minore comparsa di nuovi casi di diabete nel corso dei 4-5 anni di osservazione (RR 0,75, p < 0,001). Ciò conferisce maggiore robustezza all'azione preventiva di losartan, essendo IVS e diabete due tra i maggiori fattori di rischio cardiovascolare attualmente riconosciuti. La tollerabilità del trattamento è risultata generalmente superiore con losartan, le sospensioni della terapia a causa di effetti indesiderati correlati al farmaco più frequenti con atenololo ( $p<0,0001)$. L'insieme dei risultati conseguiti su questo campione di ipertesi è particolarmente importante, in considerazione del fatto che nessun altro trattamento si era dimostrato superiore ai beta-bloccanti o ai diuretici nel controllo di morbilità e mortalità cardiovascolari associate a pressione arteriosa elevata.

Nell' ambito dello studio LIFE sono state condotte analisi statistiche anche su sottogruppi prespecificati di pazienti, i cui dati sono stati pubblicati a parte in lavori collegati, che nel complesso hanno fornito risultati analoghi allo studio principale, confermando la superiore efficacia preventiva di losartan rispetto ad atenololo nei pazienti diabetici, con riduzione del $26 \%(\mathrm{p}<0,05)$ della frequenza end-point primario [8], e nei pazienti senza evidenza di malattia cardiovascolare all' arruolamento [9], in cui la riduzione relativa del rischio è risultata del 19\% ( $\mathrm{p}<0,001)$. In particolare il rischio relativo di ictus si è ridotto del $18 \%$ nei pazienti diabetici e del $34 \%$ in quelli senza malattie cardiovascolari all'aruolamento, in confronto ai pazienti trattati con atenololo.

Lavori preliminari ed esperimenti condotti con gli ACE-inibitori indicano la possibilità che il blocco del sistema renina-angiotensinaaldosterone sia in grado di rallentare la progressione del danno renale dovuta a diabete e/o ipertensione al di là degli effetti sulla pressione arteriosa. RENAAL, un ampio studio multicentrico controllato versus placebo, è stato disegnato specificatamente per testare quest'ipotesi. 1513 pazienti affetti da diabete di tipo II e nefropatia sono stati arruolati e assegnati al trattamento con losartan o placebo, associato a terapia antipertensiva convenzionale (con esclusione di ACE-inibitori e ARB) nei soggetti che la richiedessero per raggiungere valori pressori inferiori a 140/90 $\mathrm{mmHg}$ [10]. La durata media del follow-up è stata di 3,4 anni; l'end-point di valutazione primario era costituito da un parametro combinato, composto da raddoppio dei valori di creatininemia, insufficienza renale allo stadio terminale o morte per qualsiasi causa. I pazienti assegnati al losartan (100 mg/die per il $71 \%$ del campione) 
hanno mostrato un rischio di occorrenza dell'end-point primario ridotto del $16 \%$, diminuzione sostanzialmente data dalle componenti renali del parametro composito. Il risultato più significativo riguarda infatti la differenza osservata tra gruppi in merito al rischio di sviluppare nefropatia terminale, inferiore del $28 \%$ in 3,4 anni di trattamento (Figura 2).

Gli autori del lavoro, estrapolando da tale valore, hanno stimato che la protezione renale osservata corrisponderebbe a un rinvio medio di due anni della necessità di ricorrere alla dialisi o al trapianto renale. Per quanto la pressione media sia risultata leggermente inferiore nel gruppo losartan, la riduzione della progressione del danno d'organo è stata ampiamente superiore a quella attribuibile a tale differenza, dimostrando l'ipotesi che costituiva il razionale dello studio. I pazienti nei due gruppi hanno avuto valori non significativamente diversi di mortalità e morbilità cardiovascolare e di mortalità per qualsiasi causa; la comparsa di eventi avversi che hanno portato alla sospensione del trattamento è occorsa nel 17,2\% dei pazienti assegnati al farmaco attivo e nel $21,7 \%$ dei controlli.

\section{TOLLERABILITÀ}

I dati sulla sicurezza raccolti nel corso dei grandi trial clinici descritti sopra indicano che l'assunzione di losartan è associata a una frequenza complessiva di effetti indesiderati simile a quella osservata con il placebo, tanto che la sospensione della terapia è risultata più frequente nei controlli $(3,7 \%)$ che nei soggetti esposti al farmaco attivo $(2,3 \%)$ [11].

L'incidenza degli effetti avversi correlati al losartan non sembra essere dose-dipendente ed è simile nei vari gruppi di età e sesso. Negli oltre 3300 pazienti ipertesi che costituiscono la casistica esaminata per la definizione della tollerabilità del losartan a fini registrativi, gli eventi avversi registrati con maggiore frequenza sono stati la cefalea (14\%), l'infezione delle vie aeree superiori $(7,9 \%)$, il senso di stordimento $(3,5 \%)$ e la tosse $(3,4 \%)$. La bassa frequenza e la modesta gravità degli effetti collaterali sono alla base della buona compliance che caratterizza l'assunzione di losartan.

Un altro aspetto correlato, in quanto in grado di facilitare l'aderenza al trattamento, è la maneggevolezza di losartan e degli ARB in generale. In questo senso, maneggevolezza è da intendersi come assenza di interazioni farmacologiche importanti, in particolare con farmaci frequentemente prescritti nei pazienti ipertesi. La Tabella 2 confronta il profilo delle interazioni degli ACE-inibitori con quella degli ARB.

\section{ANALISIFARMACOECONOMICHE}

Gli studi farmacoeconomici si prefiggono di rendere manifeste le conseguenze complessive delle scelte in campo sanitario, evidenziandone i risvolti economici e clinici e mettendoli in relazione tra loro.

\begin{tabular}{lll}
\hline & \multicolumn{1}{c}{ ACE-I } & \multicolumn{1}{c}{ ARB } \\
\hline FANS & Riduzione efficacia (2) & \\
Allopurinolo & Reazioni di ipersensibilità (3) & \\
Azatioprina & Rischio di mielosoppressione (3) & \\
Ciclosporina & Rischio di nefrotossicità (2) \\
Diuretici & Ipotensione ortostatica (2) & \\
EPO & Riduzione efficacia EPO (2) & \\
IFN alfa-2 & Tossicità ematologica (3) & \\
Metformina & Tossicità ematologica (2) & Tossicità da litio (3) \\
Litio & Tossicità da litio (2) & Iperkaliemia (1) \\
Potassio & Iperkaliemia (3) & \\
Rifampicina & Riduzione efficacia (1) & Riduzione efficacia (2) \\
Ritonavir & Riduzione efficacia (1) & \\
Tetraciclina & & \\
\hline
\end{tabular}

Tabella 2

Interazioni farmacologiche di ACE-I e ARB Legenda: 1 Interazione minore; 2 Interazione moderata; 3 Interazione Maggiore 
Losartan, come gli altri ARB e in generale $i$ farmaci innovativi, ha un costo di acquisto superiore a quello della maggioranza degli altri antipertensivi. D'altro canto, possiede alcune caratteristiche farmacologiche e cliniche potenzialmente in grado di compensare l'eccesso di spesa legato al costo farmaceutico: l'efficacia antipertensiva, la protezione degli organi bersaglio, la buona compliance. I lavori pubblicati nella letteratura specializzata si sono concentrati su questi ultimi due aspetti.

L'obiettivo primario del trattamento dell'ipertensione non è tanto la diminuzione della pressione sanguigna in sé quanto la riduzione del rischio cardiovascolare, associato in maniera diretta e lineare ai valori pressori: gli studi condotti negli ultimi trent' anni hanno dimostrato un netto calo degli eventi cardiovascolari e delle loro complicazioni in caso di buon controllo pressorio. Coerentemente con tali evidenze, i farmaci antipertensivi sono rimborsati dai sistemi sanitari pubblici, che hanno riconosciuto come la riduzione a lungo termine dei costi clinici, sociali ed economici dell'ipertensione giustifichi ampiamente l'investimento. Sfortunatamente, i dati indicano che il controllo della pressione arteriosa nella popolazione è tutt'altro che ottimale: la prevalenza di ipertensione oscilla tra il 30 e il $75 \%$, a seconda della fascia di età presa in considerazione, di cui quasi la metà non è consapevole della sua condizione e non segue una terapia; tra i pazienti trattati, non più del 30-40\% raggiunge i valori auspicabili per ottenere la massima riduzione del rischio globale [12].

Le molteplici ragioni di tale scarso controllo sono state recentemente analizzate in maniera dettagliata da Mancia e coll. [13], che hanno individuato ragioni economiche, pratiche e cliniche. La scarsa consapevolezza dei rischi legati all'elevata pressione e dei benefici del trattamento, dovuta a insufficiente comunicazione da parte del medico o incomprensione da parte del paziente è risultata uno dei principali fattori. Un ulteriore ruolo viene ricoperto dalla complessità dello schema posologico, con dimostrazioni del progressivo miglioramento dell'aderenza al trattamento in corrispondenza della riduzione di compresse da assumere nell'arco della giornata.

Le ragioni preminenti dell'abbandono del regime terapeutico in vari studi esaminati da Mancia e coll. [13] sono comunque state l'inadeguatezza del controllo pressorio raggiunto e la presenza di effetti indesiderati. Ciò, continuano gli autori, mette in luce il fatto che la tollerabilità di un farmaco antipertensivo è caratteristica almeno altrettanto importante della sua efficacia, anche in virtù del fatto che la malattia ipertensiva è abitualmente asintomatica fino alla comparsa delle complicazioni cardiovascolari: la compromissione della qualità di vita in occorrenza di eventi indesiderati è considerata più rilevante dei rischi futuri, in particolare nell'evenienza, frequente, che non sia ben compreso il pericolo associato all'ipertensione, ancorché silente. L'interruzione del trattamento antipertensivo rappresenta un doppio fallimento, clinico ed economico. Dal punto di vista economico è una perdita perché i soldi spesi per l'acquisto del farmaco costituiscono un investimento a perdere, perché aumenta la necessità di ulteriori visite e prescrizioni, e perché si perdono i benefici futuri, intesi come eventi cardiovascolari e premorienza (e relativi costi) evitati.

La compliance alla terapia con losartan non è risultata migliore dei farmaci concorrenti solo in condizioni sperimentali (studio LIFE), ma è dimostrata anche nella pratica quotidiana. Dati raccolti dal servizio di farmacovigilanza britannico, ad esempio, hanno indicato che 1' $82,5 \%$ di oltre 14.000 pazienti in cura presso medici di medicina generale tolleravano bene la terapia cronica con losartan [14]. In uno studio prospettico condotto da Bloom [15], la persistenza in terapia a 12 mesi dei pazienti cui era stato inizialmente prescritto losartan è risultata significativamente superiore a quella dei pazienti trattati con ACE-inibitori, calcioantagonisti, beta-bloccanti e diuretici tiazidici. Dopo 48 mesi di follow-up, nonostante un progressivo decremento dei valori in tutti i gruppi, la tendenza è rimasta la medesima.

Risultati analoghi sono stati ottenuti in un altro lavoro di Conlin e coll.[16], che ha inoltre dimostrato come la percentuale di pazienti persistenti tra i 12 e i 48 mesi dall'inizio della terapia fosse superiore con losartan che con qualsiasi altra classe di antipertensivi $(\mathrm{p}<0,001)$.

Per quanto riguarda l'Italia, la persistenza nel trattamento farmacologico dell'ipertensione e i suoi risvolti economici sono stati analizzati dal gruppo di Degli Esposti su dati ottenuti dal database dell' ASL di Ravenna relativo all'anno solare 1997 [17]. La popolazione che rispondeva ai criteri di inclusione comprendeva quasi 17 mila pazienti ipertesi; di questi, ben il $65 \%$ ha interrotto ogni trattamento antipertensivo nel corso dell' anno di osservazione, 1 ' $8 \%$ lo ha cambiato e solo il $27 \%$ ha proseguito con la terapia prescritta inizialmente. Tra i fattori predittivi di persistenza, sono risultati associati in maniera statisticamente significativa alcuni parametri legati al paziente (età all'arruolamento, presenza/assenza di cardiopatia, diabete, asma) e la classe di antipertensivi prescritta. In relazione al farma- 
co scelto, la persistenza variava da un minimo del $30 \%$, osservato con i diuretici, a un massimo di $58 \%$ ( $\mathrm{p}<0,0001$ per la differenza rispetto a qualsiasi altra classe) con gli ARB, con risultati intermedi per beta-bloccanti, calcio-antagonisti e ACE-inibitori, in ordine crescente. Tradotto in termini di rischio relativo, tale dato indica che, a parità di tutte le altre condizioni analizzate, i pazienti che assumevano farmaci diversi dagli ARB avevano oltre il 50\% di possibilità in più di interrompere la terapia nel corso dell'anno esaminato. L'analisi condotta in questo studio non si ferma però al dato puro dei pazienti che continuano, cambiano o interrompono la terapia, ma include una valutazione dei costi. La spesa complessiva annuale per farmaci antipertensivi nei pazienti analizzati superava il milione di euro, di cui il $25 \%$ è stato investito per la cura dei pazienti non persistenti. Premesso che la spesa sostenuta per questi pazienti risulta essere un investimento inefficiente, in quanto non riduce il rischio cardiovascolare (oltre l' $80 \%$ dei non persistenti ha interrotto il trattamento dopo la prima prescrizione), gli autori hanno costruito un indice di efficienza dell'investimento per le singole classi farmacologiche, corrispondente al rapporto tra la spesa farmaceutica destinata ai pazienti persistenti e la spesa totale di quella classe. A prescindere dal costo della terapia gli ARB essi mostrano comunque il maggiore indice di efficienza tra le opzioni disponibili (Figura 3).

È inoltre parzialmente fuorviante considerare le varie classi farmacologiche come alternative a sé stanti, poiché la maggioranza dei pazienti richiede comunque una terapia di combinazione per raggiungere gli obiettivi pressori. La pratica clinica e i dati sperimentali indicano che è più agevole mantenere un buon profilo di tollerabilità, coniugato con importanti riduzioni pressorie, mediante l'uso di una combinazione di farmaci che con dosi elevate di un singolo agente; in quest'ottica l'aggiunta al regime terapeutico di un farmaco efficace e ben tollerato rappresenta una possibilità da sfruttare per massimizzare i benefici sanitari di un paziente senza rischiare di "perderlo", a causa della riduzione di qualità di vita indotta dagli effetti collaterali.

L'altro grosso filone di ricerca farmacoeconomica sul losartan si è rivolto soprattutto a valorizzare in termini monetari gli effetti renoprotettivi evidenziati dallo studio RENAAL. Gli stessi ricercatori che hanno condotto lo studio hanno valutato gli effetti economici (limitati alla durata del trial) del rallentamento della progressione della nefropatia indotto dall'aggiunta di losartan alla terapia antipertensiva convenzionale [18]. A tal fine, hanno calcolato il numero di giorni di IRC (insufficienza renale cronica) evitati nel corso dei 3,5 anni dello studio per differenza tra l'area sotto le curve di Kaplan-Meier relative alla sopravvivenza libera da malattia e alla sopravvivenza assoluta, risultati uguali a 33,6 per paziente. La valorizzazione del costo per giornata di IRC è avvenuta sulla base dei costi per paziente diabetico dializzato contenuti nei dati dell'U.S. Renal Data System e tiene conto della riduzione delle spese dopo i primi 90 giorni dall'inizio dell'emodialisi. Il rallentamento della progressione della nefropatia diabetica risulta in una riduzione dei costi dell' IRC uguale a 5.144 USD per paziente, cui sono stati sottratti i costi farmaceutici del losartan, con un risparmio netto di 3.522 USD nel corso di 3,5 anni e, per estrapolazione, di 5.298 USD in 4 anni. In altri termini, ciò significa che per ogni dollaro investito in losartan in pazienti diabetici dalle caratteristiche simili a quelle dello studio, si realizzano 3,17 dollari di risparmio in risorse sanitarie destinate alla cura dell' IRC. La riduzione dei costi dell'IRC inizia dopo circa 1,5 anni di terapia, per cui l'andamento dei costi totali presenta una fase iniziale nei quali i costi

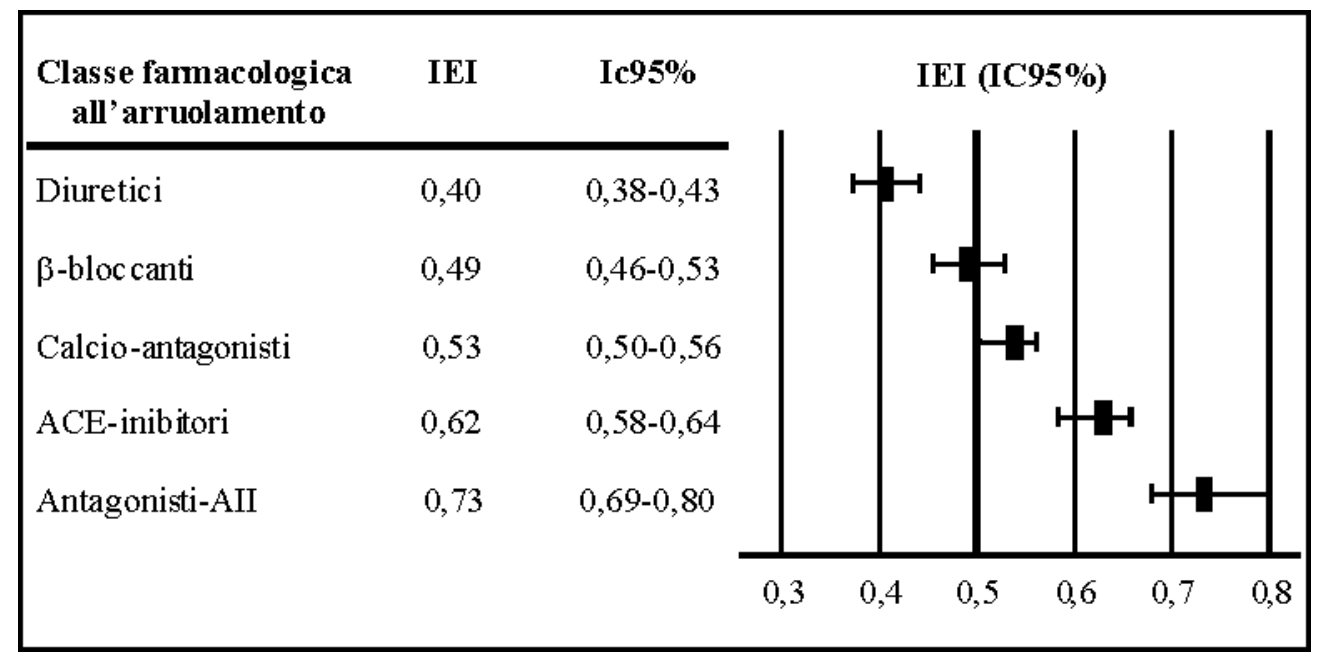

Figura 3

Indice di efficienza [17] 
del farmaco superano i risparmi, per raggiungere il pareggio economico dopo circa due anni, dopo i quali i risparmi aumentano costantemente fino alla fine dei 4 anni presi in considerazione. (Figura 4)

Sandoz e coll. [19] hanno condotto uno studio simile, applicato al contesto sanitario svizzero. La maggiore differenza rispetto al lavoro precedente consiste, oltre che nei differenti costi del farmaco e degli interventi sanitari, nell'avere valorizzato i giorni di IRC secondo il mix di trattamento dei nefropatici cronici in Svizzera: $80 \%$ emodialisi, $14 \%$ dialisi peritoneale e $6 \%$ trapianto renale. I risultati ottenuti sono analoghi a quelli americani, con un risparmio netto stimato in 4.423 franchi per paziente e conseguimento del pareggio finanziario nell'intervallo compreso tra i 2 e i 2,5 anni dall'inizio della terapia.

Il medesimo approccio è stato utilizzato da Souchet e coll. [20] per i diabetici nefropatici assistiti dal servizio sanitario francese, con risultati sostanzialmente sovrapponibili: a fronte di un aumento medio di spesa farmaceutica per paziente di 1.603 euro in 4 anni, è stato stimato un risparmio netto medio sui costi totali pari a 5.835 euro per paziente.

Nella discussione dei loro risultati, i vari autori fanno alcune considerazioni che vale la pena di riassumere brevemente, anche perché sono comuni ai vari sistemi sanitari analizzati e rimangono validi anche nel contesto italiano. Innanzitutto, la prospettiva adottata nei loro studi è quella del terzo pagante (assicurazione o servizio sanitario), per cui il risparmio calcolato non tiene conto dei costi diretti non sanitari (trasporto al servizio di dialisi), dei costi indiretti (perdita di produttività del paziente e dei famigliari) e dei costi intangibili (sofferenze, perdita di qualità di vita); il guadagno economico è dunque sottostimato se si assume il punto di vista della società nel suo complesso.

Un' altra questione affrontata dagli autori è l'evoluzione dei costi dopo i quattro anni presi in considerazione; premesso che la valutazione delle conseguenze economiche suc- cessive al periodo di sperimentazione non rientrava negli obiettivi dei loro lavori, questi autori osservano che la tendenza all'aumento dei risparmi nel periodo compreso tra i 2 e i 4 anni suggerisce che le ricadute economiche nel lungo periodo vengano mantenute, se non ulteriormente incrementate.

I risultati di queste valutazioni economiche condotte sui dati RENAAL sono molto significativi, per diversi ordini di ragioni. Innanzi tutto, sono molto pochi gli interventi sanitari in grado di indurre risparmi effettivi sui costi sanitari, una delle ragioni alla base degli studi di costo/efficacia. Questa capacità di indurre risparmi si ha nei casi in cui un trattamento preventivo efficace viene applicato a soggetti ad alto rischio di sviluppare eventi sanitari ad alto costo: nel caso del diabete, si ha alto rischio di sviluppare IRC, la cui terapia è molto costosa. Altra caratteristica rilevante dell' andamento dei costi analizzato è la rapidità con cui viene raggiunto il pareggio economico, in tempi compatibili con le dinamiche di gestione dei servizi sanitari.

Gerth e coll. [21] hanno stimato l'impatto economico della prevenzione secondaria con losartan nell' ambito dell'Unione europea. I dati clinici utilizzati sono sempre quelli dello studio RENAAL e sono stati applicati al complesso dei diabetici nefropatici europei (circa 700.000), con valorizzazione degli interventi sanitari in base alle tariffe tedesche (111 euro/die per IRT). La previsione risultante è che l'aggiunta al loro regime terapeutico di losartan sia in grado di determinare una riduzione di 64.400 casi di IRT in 3,5 anni, con un risparmio di 2,6 miliardi di euro.

Gli interventi sanitari possono avere risvolti parzialmente diversi in relazione al punto di vista adottato; per illustrare il complesso dei valori (clinici ed economici, positivi e negativi) a seconda dell'attore coinvolto, si è quindi sviluppata la tecnica farmacoeconomica dell'analisi dei valori. Per ogni prospettiva adottata, vengono raffigurati in una tabella gli in-

\section{Figura 4}

Valutazione economica dei risultati RENAAL: a)Andamento dei risparmi sull'IRC b) Andamento dei risparmi netti [18]

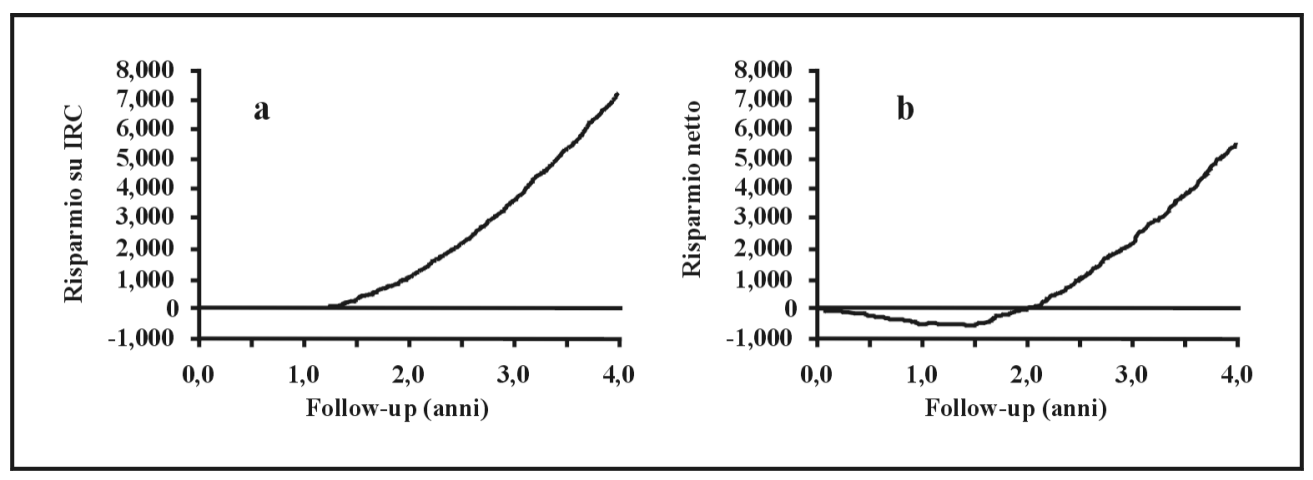




\section{Prospettiva del paziente}

\begin{tabular}{|c|c|c|}
\hline Valore & Positivo & Negativo \\
\hline linico & $\begin{array}{l}\text { Riduzione della pressione e del rischio } \\
\text { cardiovascolare associato } \\
\text { Protezione dal danno d'organo al di là } \\
\text { dell'effetto emodinamico } \\
\text { Buona tollerabilità in un'elevata } \\
\text { percentuale di pazienti } \\
\text { Riduzione della necessità di altre } \\
\text { terapie, con semplificazione dei regimi } \\
\text { farmacologici } \\
\text { Comodità della posologia (UID) }\end{array}$ & $\begin{array}{l}\text { Inefficace in alcuni pazienti } \\
\text { Necessità di associare altri farmaci nei } \\
\text { casi gravi }\end{array}$ \\
\hline conomico & $\begin{array}{l}\text { Riduzione della perdita di produttività } \\
\text { e aumento della qualità di vita del } \\
\text { paziente e del nucleo familiare }\end{array}$ & $\begin{array}{l}\text { Quote di compartecipazione della } \\
\text { spesa (variabili per regione) }\end{array}$ \\
\hline
\end{tabular}

\section{Prospettiva del medico}

Valore

Positivo

Negativo

\begin{tabular}{lll} 
Clinico & $\begin{array}{l}\text { Soddisfazione del paziente } \\
\text { Azione preventiva }\end{array}$ & $\begin{array}{l}\text { Insoddisfazione del paziente nei casi di } \\
\text { inefficacia o scarsa tollerabilità }\end{array}$ \\
& $\begin{array}{l}\text { Semplice prescrizione grazie alle } \\
\text { poche avvertenze e controindicazioni } \\
\text { Maneggevolezza per poche interazioni } \\
\text { farmacologiche }\end{array}$ & \\
Economico & $\begin{array}{l}\text { Prescrizione efficiente } \\
\text { Minori visite per buona tollerabilità }\end{array}$ & $\begin{array}{l}\text { Necessità di prescrizioni multiple nei } \\
\text { casi non controllati in monoterapia }\end{array}$ \\
\hline
\end{tabular}

\section{Prospettiva del SSN}

\begin{tabular}{lll}
\multicolumn{1}{c}{ Valore } & \multicolumn{1}{c}{ Positivo } & \multicolumn{1}{c}{ Negativo } \\
\hline Clinico & Vedi paziente e medico & $\begin{array}{l}\text { Casi di inefficacia o di scarsa } \\
\text { tollerabilità, con necessità di revisione } \\
\text { della terapia }\end{array}$ \\
Economico & $\begin{array}{l}\text { Liberazione di risorse sanitarie grazie } \\
\text { all'efficacia preventiva } \\
\text { Riduzione del fabbisogno di altri } \\
\text { farmaci rimborsati } \\
\text { Nessuna spesa per gestione tossicità }\end{array}$ & $\begin{array}{l}\text { Alto investimento iniziale in costi } \\
\text { farmaceutici }\end{array}$ \\
\hline
\end{tabular}

\section{Prospettiva della società}

\begin{tabular}{cll}
\multicolumn{1}{c}{ Valore } & \multicolumn{1}{c}{ Positivo } & \multicolumn{1}{c}{ Negativo } \\
\hline Clinico & $\begin{array}{l}\text { Miglioramento della salute e della } \\
\text { qualità di vita dei cittadini }\end{array}$ & Reazioni avverse e casi di inefficacia \\
Economico & $\begin{array}{l}\text { Aumento della produttività lavorativa } \\
\text { dei pazienti e dei loro familiari }\end{array}$ & $\begin{array}{l}\text { Costo del farmaco (vedi SSN) } \\
\text { Tale costo è probabilmente }\end{array}$ \\
& $\begin{array}{l}\text { Risparmi sul costo delle malattie } \\
\text { cardiovascolari } \\
\text { Posti di lavoro nell'industria } \\
\text { farmaceutica }\end{array}$ & $\begin{array}{l}\text { ampiamente compensato dai risparmi } \\
\text { indotti sul costo di malattia e pertanto } \\
\text { la società nel complesso ha un } \\
\text { beneficio economico netto }\end{array}$ \\
\hline
\end{tabular}

\section{Tabella 3a}

Analisi dei valori: prospettiva del paziente

\section{Tabella 3b}

Analisi dei valori: prospettiva del medico

\section{Tabella 3c}

Analisi dei valori: prospettiva del SSN

\section{Tabella 3d}

Analisi dei valori: prospettiva della società 
croci tra le valenze cliniche ed economiche: la Tabella 3 rappresenta l'analisi dei valori del losartan, sviluppata secondo le considerazioni fatte in precedenza.

\section{CONCLUSIONI}

Nonostante i risultati degli studi clinici condotti negli ultimi trent'anni e le recenti linee guida impongano una terapia aggressiva dell'ipertensione, per le rilevanti riduzioni del rischio cardiovascolare che essa ha dimostrato di indurre, nella pratica il controllo della pressione arteriosa della popolazione è insoddisfacente. Ciò comporta un elevato onere in termini di morbilità e mortalità cardiovascolare e un elevato consumo di risorse sanitarie; la spesa farmaceutica rappresenta solo una parte minore del costo di malattia complessivo dell'ipertensione [22].

Tra le ragioni dell'alto tasso di fallimento della terapia antipertensiva gioca un ruolo preminente la comparsa di effetti collaterali in grado di peggiorare la qualità di vita del paziente. Vari studi hanno indicato che losartan e gli altri ARB, in funzione della loro tollerabilità paragonabile al placebo e della virtuale assenza di interazioni farmacologiche importanti, sono associati a un livello di compliance alla terapia superiore a quello delle altri classi di agenti antipertensivi e possono rappresentare un'opportunità di migliorare la costo-efficacia della terapia dell'ipertensione.

Losartan ha inoltre dimostrato di determinare benefici clinici con meccanismi aggiuntivi alla riduzione della pressione, riducendo il rischio cardiovascolare globale e garantendo una protezione dal danno d'organo a livello renale, cardiaco e cerebrale. La possibilità di prevenire efficacemente eventi clinici importanti in soggetti ad alto rischio non è solo un benvenuto progresso clinico, ma ha la potenzialità di incidere sui costi sanitari complessivi delle patologie cardiovascolari. Ciò è stato dimostrato per quanto riguarda il rallentamento della progressione della nefropatia diabetica, in più contesti sanitari, ed è ipotizzabile per altre patologie ad alto impatto economico, il cui rischio viene ridotto da losartan, come gli ictus (LIFE), lo scompenso cardiaco che necessita di ricovero (ELITE, RENAAL), il deterioramento cognitivo [23], l'insorgenza di nuovi casi di diabete.

In conclusione, la spesa consistente legata al costo farmaceutico di losartan va rapportata alle caratteristiche farmacologiche cliniche che vanno ad incidere, con effetto compensatorio, sul costo complessivo della patologia cardiovascolare: riduzione efficace della pressione e del rischio cardiovascolare globale con ottima tollerabilità e maneggevolezza, in grado di aumentare l'aderenza al trattamento e di ottimizzare i benefici clinici.

\section{BIBLIOGRAFIA}

1 Lo MW, Goldberg MR, McCrea JB, Lu H, Furtek CI, Bjornsson TD. Pharmacokinetics of losartan, an angiotensin II receptor antagonist, and its active metabolite EXP3174 in humans. Clin Pharmacol Ther. 1995 Dec;58(6):641-9.

2 Weber MA. Clinical experience with the angiotensin II receptor antagonist losartan. A preliminary report. Am J Hypertens. 1992 Dec;5(12 Pt 2):247S-251S.

3 Owens P, Kelly L, Nallen R et al. Comparison of antihypertensive and metabolic effects of losartan and losartan in combination with hydrochlorothiazide - a randomized controlled trial. J Hypertens 2000; 18:339-345.

4 Lozano JV, Llisterri JL, Aznar J et al. Losartan reduces microalbuminuria in hypertensive microalbuminuric type 2 diabetics; Spanish Working Group. Nephrol Dial Transplant 2001; 16(suppl 1):85-89.

5 Pitt B, Segal R, Martinez FA et al. Randomised trial of losartan versus captopril in patients over 65 with heart failure (Evaluation of Losartan in the Elderly Study, ELITE) Lancet. 1997 Mar 15;349(9054):747-52.

6 Pitt B, Poole-Wilson PA, Segal R et al. Effect of losartan compared with captopril on mortality in patients with symptomatic heart failure: randomised trial-the Losartan Heart Failure Survival Study ELITE II. Lancet. 2000 May 6;355(9215):1582-7.

7 Dahlof B, Devereux RB, Kjeldsen SE et al. Cardiovascular morbidity and mortality in the Losartan Intervention For Endpoint reduction in hypertension study (LIFE): a randomised trial against atenolol; the LIFE Study Group. Lancet 2002; 359:995-1003.

8 Lindholm LH, Ibsen H, Dahlof B et al. Cardiovascular morbidity and mortality in patients with diabetes in the Losartan Intervention For Endpoint reduction in hypertension study (LIFE): a randomised trial against atenolol. Lancet. 2002; 359(9311):1004-10. 
9 Devereux RB, Dahlof B, Kjeldsen SE et al. Effects of losartan or atenolol in hypertensive patients without clinically evident vascular disease: a substudy of the LIFE randomized trial. Ann Intern Med. 2003 Aug 5;139(3):169-77.

10 Brenner BM, Cooper ME, de Zeeuw D et al.; RENAAL Study Investigators. Effects of losartan on renal and cardiovascular outcomes in patients with type 2 diabetes and nephropathy. N Engl J Med. 2001 Sep 20;345(12):861-9.

11 Product Information: Cozaar(R), losartan potassium. Merck \& Co., Whitehouse Station, NJ (PI revised 8/2002) reviewed 12/2002.

12 Hajjar I, Kotchen TA Trends in prevalence, awareness, treatment, and control of hypertension in the United States, 1988-2000. JAMA. 2003 Jul 9;290(2):199-206.

13 Mancia G, Seravalle G, Grassi G. Tolerability and treatment compliance with angiotensin II receptor antagonists. Am J Hypertens. 2003 Dec;16(12):1066-73.

14 Mann RD, Mackay F, Pearce G et al. Losartan: a study of pharmacovigilance data on 14552 patients. J Human Hypertens 1999;13:551-7.

15 Bloom BS. Continuation of initial antihypertensive medication after 1 year of therapy. Clin Ther. 1998 JulAug;20(4):671-81.

16 Conlin PR, Gerth WC, Fox J et al. Four-Year persistence patterns among patients initiating therapy with the angiotensin II receptor antagonist losartan versus other artihypertensive drug classes. Clin Ther. 2001 Dec;23(12):1999-2010.

17 Degli Esposti L, Capone E, Degli Esposti E et al. Analisi della persistenza e delle risorse allocate nel trattamento farmacologico dell'ipertensione arteriosa. Farmeconomia e percorsi terapeutici 2002;3(3):161-70.

18 Herman WH, Shahinfar S, Carides GW et al. Losartan reduces the costs associated with diabetic end-stage renal disease: the RENAAL study economic evaluation. Diabetes Care. 2003 Mar;26(3):683-7.

19 Sandoz MS et al. Benefici medico-economici di losartan in pazienti con diabete di tipo 2 e nefropatia: valutazione economica dello studio RENAAL nel contesto sanitario svizzero. Diabetologia 2002;45(Suppl. 2):A302-3.

20 Souchet T, Durand Zaleski I, Hannedouche T et al. An economic evaluation of Losartan therapy in type 2 diabetic patients with nephropathy: an analysis of the RENAAL study adapted to France. Diabetes Metab. 2003 Feb;29(1):29-35.

21 Gerth WC et al. Losartan riduce oneri e costi della IRT: implicazioni dallo studio RENAAL per la salute pubblica nell'Unione Europea. Diabetologia 2002;45(Suppl. 2):A302.

22 McIntyre H, Costa FV, Dusing R et al. The role of losartan in cost-effective hypertension control. Curr Med Res Opin. 2002;18(3):139-45.

23 Jonsson L, Gerth W, Fastbom J. The potential economic consequences of cognitive improvement with losartan. Blood Press. 2002;11(1):46-52 